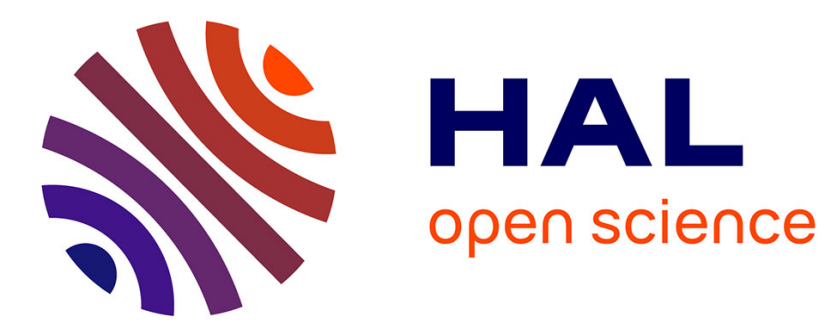

\title{
Vesicle tumbling inhibited by inertia
}

Aymen Laadhari, Pierre Saramito, Chaouqi Misbah

\section{To cite this version:}

Aymen Laadhari, Pierre Saramito, Chaouqi Misbah. Vesicle tumbling inhibited by inertia. Physics of Fluids, 2012, 24 (3), pp.031901. 10.1063/1.3690862 . hal-00604401v2

\section{HAL Id: hal-00604401 \\ https://hal.science/hal-00604401v2}

Submitted on 1 Jul 2011

HAL is a multi-disciplinary open access archive for the deposit and dissemination of scientific research documents, whether they are published or not. The documents may come from teaching and research institutions in France or abroad, or from public or private research centers.
L'archive ouverte pluridisciplinaire HAL, est destinée au dépôt et à la diffusion de documents scientifiques de niveau recherche, publiés ou non, émanant des établissements d'enseignement et de recherche français ou étrangers, des laboratoires publics ou privés. 


\title{
Vesicle tumbling inhibited by inertia
}

\author{
Aymen Laadhari, ${ }^{1}$ Pierre Saramito, ${ }^{1}$ and Chaouqi Misbah ${ }^{2}$ \\ 1) Université de Grenoble and CNRS, Lab. J. Kuntzmann (UMR 5224) F-38041 Grenoble France \\ ${ }^{2)}$ Université de Grenoble and CNRS, Lab. Interdisciplinaire de Physique (UMR 5588) F-38041 France
}

Vesicles under flow constitute a model system for the study of red blood cells (RBCs) dynamics and blood rheology. In the blood circulatory system the Reynolds number (at the scale of the RBC) is not always small enough for the Stokes limit to be valid. We develop a numerical method based on the the level set approach and solve the fluid/membrane coupling by using an adaptive finite element technique. We find that a Reynolds number of order one can destroy completely the vesicle tumbling motion obtained in the Stokes regime. We analyze in details this phenomenon and discuss some of the far reaching consequences. We suggest experimental tests on vesicles.

PACS numbers: 87.16.D- 83.50.Ha 87.17.Jj 83.80.Lz 87.19.rh

\section{INTRODUCTION}

The study of models of red blood cells (RBCs), such as capsules and vesicles, has known recently an upsurge of interest in various communities, such as physics, applied mathematics, mechanical engineering, and so on. A systematic experimental analysis ${ }^{1}$ of RBCs under shear flow has revealed that RBCs can either undergo a tanktreading (TT) or tumbling (TB) motion. In the TT regime the main axis makes a given angle (less than $\pi / 4$ ) with respect to the flow direction whereas the RBCs membrane undergoes a tank-treading motion. Upon increasing the viscosity contrast $\lambda$ (ratio of the internal over the external fluid viscosities) the RBC exhibits TB (or flipping) motion. There are two classes of model systems that are used to mimic RBCs: (i) capsules and (ii) vesicles. Capsules are made of an extensible polymer membrane which is endowed with an in-plane shear elasticity (mimicking the elasticity of the cytoskeleton of the RBCs) ${ }^{2}$. Vesicles are, like RBCs, made of an inextensible phospholipid bilayer membrane which is purely fluid (thus devoid of shear elasticity) ${ }^{3}$. The inextensible character has proven to confer to vesicles rich dynamics ${ }^{3}$, since inextensibility, triggers, even to leading order, high order nonlinearities.

An early model to understand the TT-TB transition of RBCs has been presented by Keller and Skalak (KS) ${ }^{4}$. They adopted a vesicle-like model (fluid inextensible membrane), and imposed a fixed shape (only orientation in the flow is permitted). The shape of the vesicle is assumed to be ellipsoidal for which a solution for the Stokes flow was available. KS reported that the transition depends both on the viscosity contrast $\lambda$ and on the reduced volume $v$ (the actual volume over the volume of a sphere having the same area), which is given in $2 \mathrm{D}$ (the situation in which we are interested here) by $v=(\mathcal{A} / \pi) /(\mathcal{P} / 2 \pi)^{2}$, where $\mathcal{A}$ is the area occupied by the internal liquid and $\mathcal{P}$ is the vesicle perimeter.

The analytical as well as numerical calculations ${ }^{4-6}$ for the TT-TB transition have been restricted to the Stokes limit: the inertia was neglected. Available experimental data on vesicles ${ }^{7,8}$ correspond to the very small Reynolds numbers limit. However, in the blood circulatory sys- tem, especially in the arterioles, the Reynolds number evaluated at the scale of the RBC may be of order unity. For example, in the arterioles ${ }^{9}$ the mean velocities is of about $U \sim 20 \mathrm{~cm} / \mathrm{s}$. Defining the Reynolds number as $R e=\rho_{o} R U / \eta_{o}$ where $R$ is the $\mathrm{RBC}$ radius (the radius of a sphere having the same area as the $\mathrm{RBC}$; it is equal to about $3 \mu \mathrm{m}$ for human RBCs), $\eta_{o}$ the plasma viscosity and $\rho_{o}$ its density, leading to $R e \sim 1$. From this rough estimate (see later for more precise estimate) it follows that the inertial effect is of the same order as the viscous one.

Our main objective in this study is to analyze the dynamics of vesicles under a shear flow by taking into account the inertial effects. Our study reveals that a Reynolds number of order unity is capable of inhibiting TB in favor of TT motion. We analyze the phase diagram in the relevant parameter space, and discuss some implications together with the experimental feasibility to test this prediction.

\section{MODEL AND TECHNIQUES}
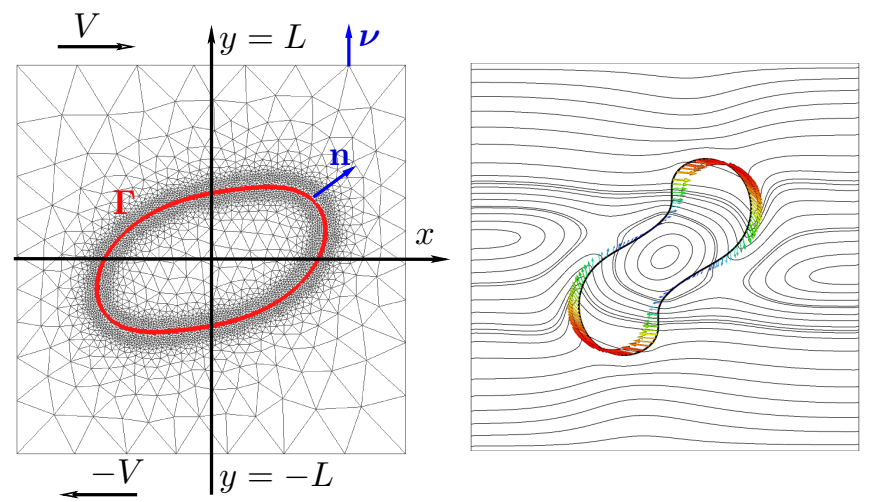

Figure 1. (color on-line) The set-up and notations. Actually the vesicle shapes, in the TB regime, shown here are obtained by numerical simulations. Left: a vesicle with a weak inertia $(R e=0.01)$. Right: a vesicle with inertia $(R e=10)$ showing ample vesicle deformation as compared to that in the Stokes limit. The finite element network and the flow lines are shown. 
We consider a $2 \mathrm{D}$ vesicle under a linear shear flow. The legitimacy of a $2 \mathrm{D}$ geometry is supported by the fact that the $2 \mathrm{D}$ dynamics obtained in numerical simulations ${ }^{6}$ are in good agreement with results obtained in 3D by KS. Hereafter $\mathbf{r}$ will denote a two dimensional position vector having the Cartesian components $x$, along the plates, and $y$, in the perpendicular direction (see Fig. 1). The vesicle is immersed in a fluid occupying a square domain of lateral length $2 L$. Let $R$ be the radius of a circle having the same perimeter as the vesicle. The aspect ratio is given by $R / L$.

The velocity and pressure fields obey in the two fluid domains (inside and outside the vesicle)

$$
\begin{array}{r}
\rho\left(\frac{\partial \mathbf{u}}{\partial t}+\mathbf{u} \cdot \nabla \mathbf{u}\right)-\operatorname{div}(2 \eta D(\mathbf{u}))+\nabla p=0 \\
\operatorname{div} \mathbf{u}=0
\end{array}
$$

where $D(\mathbf{u})=\left(\nabla \mathbf{u}+\nabla \mathbf{u}^{\mathrm{T}}\right) / 2$ is the deformation rate tensor, with $\eta=\eta_{i}$ is the internal viscosity and $\eta=\eta_{o}$ is the external one. At the vesicle membrane the hydrodynamic stress is balanced by the the membrane force

$-\kappa\left(\frac{\partial^{2} H}{\partial s^{2}}+\frac{H^{3}}{2}\right) \mathbf{n}+H \zeta \mathbf{n}-\frac{\partial \zeta}{\partial s} \mathbf{t}+[2 \eta D(\mathbf{u})-p I] \mathbf{n}=0$

where $\kappa$ is the membrane bending rigidity, $H$ is the curvature, $\mathbf{n}$ and $\mathbf{t}$ are the normal and tangential unit vectors, and $\zeta$ is a Lagrange multiplier enforcing locally constant arclength. It is fixed by requiring the surface divergence of the velocity field to vanish

$$
\operatorname{div}_{s} \mathbf{u}=0
$$

The two plates at $y= \pm L$ move horizontally with opposite constant velocity $V$ (shear flow). At $y= \pm L$, we impose no-slip condition $\mathbf{u}=( \pm V, 0)$, while on the lateral size $x= \pm L$ we impose stress-free surface (see Fig. 1 ).

The Navier-Stokes equations are nonlinear, so that the quite precise method based on boundary integral formulation $^{6,10}$ can not be used. We resort here to the level set approach. Very briefly, in this method the membrane location is defined by a level set function $\phi(\mathbf{r}, t)$ depending on the $2 \mathrm{D}$ vector $\mathbf{r}=(x, y)$ and time. The membrane position is taken to be (this is an implicit representation of the membrane) $\phi=0$, and the level set function obeys a transport equation

$$
\frac{\partial \phi}{\partial t}+\mathbf{u} \cdot \nabla \phi=0
$$

Because $\phi$ is defined everywhere in the entire domain, the fluid/membrane interaction is solved in a fully Eulerian scheme. The normal and the tangent vectors, as well as the curvature are defined in terms of $\phi: \mathbf{n}=\nabla \phi /|\nabla \phi|$, $H=\operatorname{div} \mathbf{n}$, so that the full membrane force can be defined in the entire domain. The Lagrange multiplier is also defined in the entire domain. Note that while the membrane force is defined in the whole domain, its action is localized to the membrane region only.
The above set of equations has been reformulated in terms of a variational representation (the so-called weak formulation) and has been implemented in a finite element scheme. We use an improvement of the classical level set method that takes care of solving exactly the volume and area constraints. Details of the numerical study and benchmark tests are published elsewhere ${ }^{11}$, while we focus here on the main physical results only.

We adimensionalize the equations by choosing $R$ as a length scale, $U=V R / L$ as a velocity scale, $T=R / U$ as a unit of time and $\eta_{o} U / R$ as a unit of pressure. This leads us to three dimensionless physical parameters

$$
R e=\frac{\rho_{o} V R^{2}}{\eta_{o}}, \quad C a=\frac{\eta_{o} R^{3} \dot{\gamma}}{\kappa}, \quad \lambda=\frac{\eta_{i}}{\eta_{o}}
$$

This set has to be supplemented with two geometrical parameters, namely the vesicle confinement $C n=R / L$ and the reduced area $v$.

Here we shall take the same densities inside and outside the vesicle, and we shall keep the confinement to a given value (typically 0.4 ). Moreover, in $2 \mathrm{D}$, the TT-TB transition is quasi-insensitive ${ }^{6}$ to $C a$. We set $C a=100$, and have checked that higher or smaller values do not affect the results. We are thus left with three free parameters $v, \lambda$ and $R e$. In the exploration of the inertial effect, we shall prescribe a given value for $v$ and vary only $\lambda$ and $R e$. However, in order to compare our results obtained in the quasi-Stokes regime to existing numerical results in the Stokes regime we have also examined several values of $C n$ and $v$, as described below.

\section{RESULTS AND DISCUSSION}

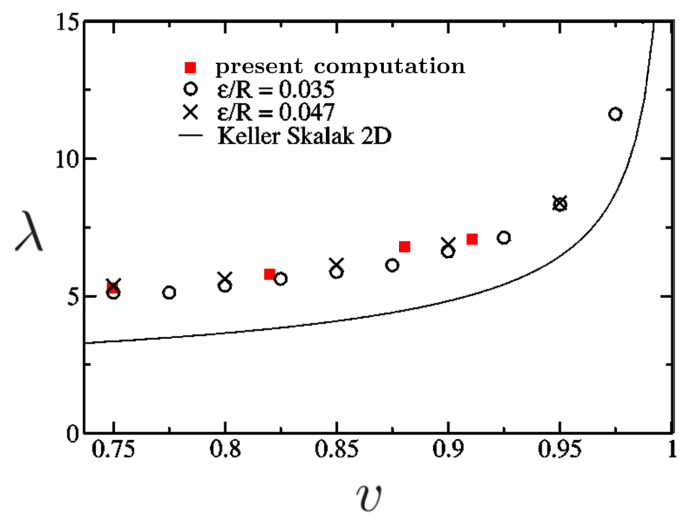

Figure 2. Phase diagram and comparison with the phase field method $^{6}$ (circles and crosses) where $\varepsilon$ denotes the interface width.

In order to test and validate our analysis, we compare our results with available numerical data and set $R e=$ $10^{-2}$. In our computations, a small confinement $C n=$ 0.25 has been chosen, so that the influence of boundaries be weak enough (see also $\left.{ }^{11}\right)$. We have varied $\lambda, v$ and 
have determined the transition line separating the regime of tank-treading (TT) from that of tumbling (TB). The results are presented in Fig. 2 showing a good agreement with the phase field method (see Fig. 5 in Ref. ${ }^{6}$ ). Remark that the $2 \mathrm{D} \mathrm{KS}$ theory ${ }^{4}$, based on some simplification hypothesis, is able to capture the main behavior of the phase diagram.
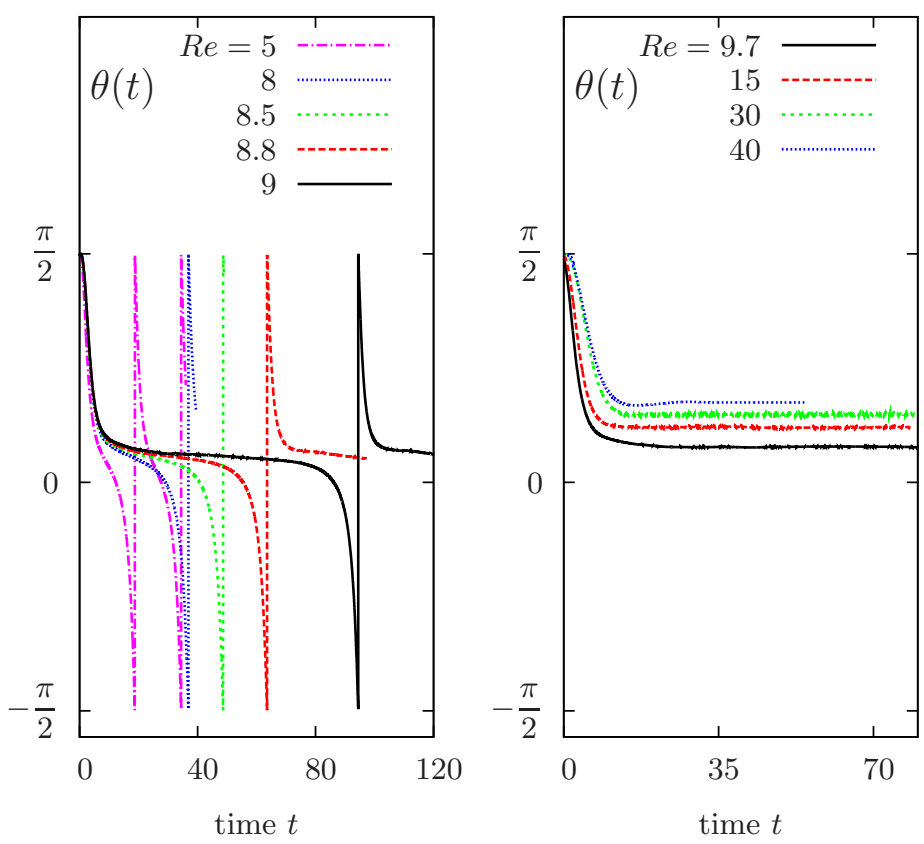

Figure 3. The behavior of the angle of the main axis of the vesicle as a function of time for different Reynolds numbers, showing that inertia slows down the motion, until suppressing TB $(v=0.82, \lambda=20)$.

Next we examine the role of the Reynolds number on the two dynamical regime TT and TB. A first noticeable effect is that upon increasing $R e$ in the TB regime the period of oscillation increases significantly (Fig. 3.a and 4) until it diverges (Fig. 3.b) for a critical value of $R e$. The interesting fact is that this behavior occurs for quite moderate values of $R e$. The divergence of the period means that the TB is suppressed in favor of a TT regime. Another impact of the inertial effect is that the vesicle adopts in the TT regime a terminal angle that significantly depends on $R e$; the terminal angle can have values which may be twice as large as compared to those obtained in the Stokes regime (see Fig. 5). Furthermore, the effect of inertia causes a stronger deformation of the vesicle, as shown on Fig. 1.

Our current intuitive understanding is as follows. In the absence of inertia TB occurs ${ }^{5}$ when the viscosity contrast reaches a critical value such that the torque due to the applied shear flow can not anymore be efficiently converted into the membrane tank-treading torque because the internal fluid is so viscous that it precludes tanktreading. Therefore the vesicle behaves as almost quasirigid, and $\mathrm{TB}$ takes place. In other words, in the TB regime, the injected power due to shear is predominantly

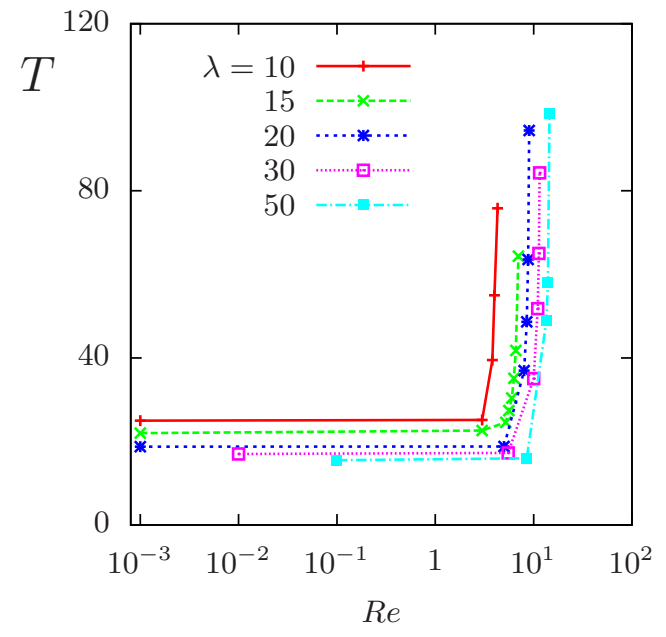

Figure 4. The behavior of the TB period $T$ vs $R e$ for various viscosity ratio $\lambda$, showing divergence of the period at a critical Reynolds number.

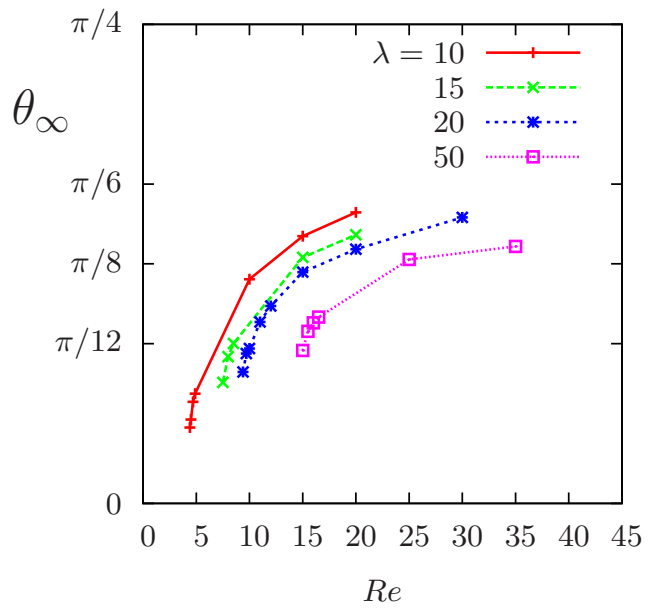

Figure 5. The behavior of the terminal angle $\theta_{\infty}$ in the TT regime vs $R e$ for various viscosity ratio $\lambda$.

transferred to dissipation of the surrounding fluid. TB in the presence of inertia must, besides dissipation in the surrounding fluid, be accompanied with kinetic energy transfer to the surrounding fluid, a cost that increases with Reynolds number so that TB becomes unfavorable.

We have performed a systematic analysis (at fixed reduced area $v=0.82$ ) on the occurrence of TT and TB as a function of the viscosity contrast $\lambda$ and Reynolds number Re. The results are reported on Fig. 6. In the absence of inertia $(R e=0)$ the TT-TB bifurcation occurs at about $\lambda=6$. As $R e$ is increased TB is delayed. For example, at $R e \simeq 5$ the critical $\lambda$ has approximately doubled (it is about 12). It is interesting to note that the separation line in Fig. 6 continues to increase, but still for $R e \sim 16$, TT continues to prevail even though the viscosity contrast is quite large $(\lambda=80)$. At such a large contrast one might be tempted to expect the vesicle to 
behave like a rigid particle (meaning that TB prevails). However, inertia is still capable of enforcing membrane tank-treading, enabling the vesicle to enjoy its fluidity, making it quite distinct from a rigid particle.

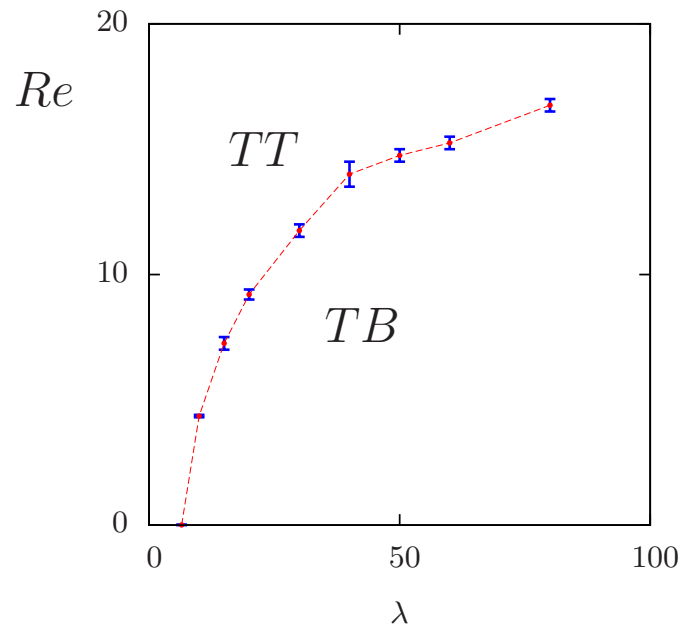

Figure 6. The phase diagram of the TT and TB motion as a function of the viscosity contrast $\lambda$ and the Reynolds numbers $(v=0.82, C a=100, C n=0.5)$.

Some concluding remarks might be useful. In human arterioles $^{9}$ the wall shear rate is of about 8000 $\mathrm{s}^{-1}$. Using the Reynolds number definition adopted here, $R e=\frac{\rho_{o} V R^{2}}{\eta_{o}}$, with $R \simeq 3 \mu \mathrm{m}$, and $\eta_{o} / \rho_{o} \simeq 10^{-2} \mathrm{~cm}^{2} / \mathrm{s}$ (plasma kinematic viscosity), one obtains $R e \sim 0.1$. When geometry suddenly changes (bifurcations, etc.) velocity gradients are enhanced resulting in much higher transient inertial effects. Furthermore, it is believed that the viscosity contrast of RBCs in vivo ${ }^{4}$ is close to (in the Stokes limit) the critical value for the TT-TB transition. This idea would mean that inhibition of TB should be favored by moderate inertial effects $(R e \sim 1)$.

Why should it be beneficial for RBCs to perform TT rather than TB at all? Our speculation is as follows. Under an alteration of the flow due, for example, to a tissue injury, or caused by abnormal widening of blood vessels (the so-called sites of aneurysms), RBCs tend to scrape along the blood vessel lining. In the TT regime cells experience a lift force of hydrodynamical (viscous) nature $8,12,13$. This force is essential to keep cells away from potential undesirable adhesion. However, RBCs which undergo TB experience practically no lift force (due to the quasi-up-down symmetry over a period of TB $)^{14}$. Transient suppression of TB (at special sites undergoing sudden changes of flow condition, where inertial effect are transiently amplified) would enable RBCs to be efficiently pushed away from blood vessel walls. A systematic study dealing with $3 \mathrm{D}$ simulations including shear elasticity (to mimic the RBC cytoskeleton) is an essential step in order to be more quantitative.

Vesicles would be a more adequate system in order to test the present finding, but also to check the above speculations. Vesicles may be produced with large enough size, for example, with radius in the range $20-40 \mu \mathrm{m}$. Imposing wall shear rates of about $8000 \mathrm{~s}^{-1}$ one finds $R e \simeq 3-15$, where we expect suppression of TB to be quite favorable. In a microfluidic channel of diameter $d \sim 100 \mu \mathrm{m}$, with maximum velocity $V_{\max }$ of the imposed Poiseuille flow, the wall shear rate is equal to $8 V_{\max } / d$. Imposing a maximum velocity of order 10 $\mathrm{cm} / \mathrm{s}$ (for which high speed camera are well adapted to capture vesicle dynamics with a good precision), one easily obtains shear rates of the order of $10^{4} \mathrm{~s}^{-1}$.

C.M. and A.L. Acknowledge financial support from CNES and ANR (MOSICOB project).

\section{REFERENCES}

${ }^{1}$ T. Fischer, M. Stohr-Lissen, and H. Schmid-Schonbein. The red cell as a fluid droplet: tank tread-like motion of the human erythrocyte membrane in shear flow. Science, 202:894-896, 1978.

${ }^{2}$ D. Barthès-Biesel. Capsule motion in flow: deformation and membrane buckling. C. R. Physique, 10(8):764-774, 2009.

${ }^{3}$ P. M. Vlahovska, T. Podgorski, and C. Misbah. Vesicles and red blood cells in flow: from individual dynamics to rheology. $C$. $R$. Physique, 10:775-789, 2009.

${ }^{4}$ S. Keller and R. Skalak. Motion of a tank-treading ellipsoidal particle in a shear flow. J. Fluid Mech., 120:27-47, 1982.

${ }^{5}$ F. Rioual, T. Biben, and C. Misbah. Analytical analysis of a vesicle tumbling under a shear flow. Phys. Rev. E, 69:061914, 2004.

${ }^{6}$ J. Beaucourt, F. Rioual, T. Seon, T. Biben, and C. Misbah. Steady to unsteady dynamics of a vesicle in a flow. Phys. Rev. E, 69:011906, 2004.

${ }^{7}$ V. Kantsler and V. Steinberg. Transition to tumbling and two regimes of tumbling motion of a vesicle in shear flow. Phys. Rev. Lett., 96:036001, 2006.

${ }^{8}$ M.-A. Mader, V. Vitkova, M. Abkarian, A. Viallat, and T. Podgorski. Dynamics of viscous vesicles in shear flow. Eur. Phys. J. E, 19:389-397, 2006.

${ }^{9}$ Y. C. Fung. Biomechanics. Springer, 1990.

${ }^{10}$ S. K. Veerapaneni, R. Raj, G. Biros, and P. K. Purohit. Analytical and numerical solutions for shapes of quiescent twodimensional vesicles. Int. J. Nonlinear Mech., 44(3):257-262, 2009.

${ }^{11}$ A. Laadhari, P. Saramito, and C. Misbah. Computing the dynamics of biomembranes by combining conservative level set and adaptive finite element methods. submitted, 2011. http://hal .archives-ouvertes.fr/hal-00604145.

${ }^{12}$ U. Seifert. Hydrodynamic lift on bound vesicles. Phys. Rev. Lett., 83(4):876-879, Jul 1999.

${ }^{13}$ I. Cantat and C. Misbah. Lift force and dynamical unbinding of adhering vesicles under shear flow. Phys. Rev. Lett., 83(4):880883, 1999.

${ }^{14}$ G. Danker, P. M. Vlahovska, and C. Misbah. Vesicles in Poiseuille flow. Phys. Rev. Lett., 102(14):148102, 2009. 